\title{
基于氨硼烷的直接转移氢化反应研究进展
}

\author{
阳香华* 谢珍茗何军余林* \\ ( ${ }^{a}$ 广东工业大学轻工化工学院 广州 510006)
}

\begin{abstract}
摘要 直接转移氢化反应是指氢原子在没有催化剂参与的情况下从氢供体转移到氢受体的过程一一即所谓不含金属 的反应. 该过程是机理研究中理想的基本步骤，却通常因反应能垒太高而鲜少报道. 然而，理论和实验研究都表明，提 高底物的极性可有效降低反应能垒，意味着极性的氢供体和氢受体直接很可能发生这类反应. 氨硼烷是一种含氢量很 高的知名化学储氢材料, 同时, 它也是一种同时含有正、负电性氢原子的理想极性氢供体. 事实上, 以氨硼烷为极性氢 供体，亚胺、极化烯烃以及醛酮等为极性氢受体，直接转移氢化反应能在温和条件下发生. 主要对这些反应的底物范围 以及机理上的异同进行了综述.
\end{abstract}

关键词 氨硼烷; 直接转移氢化反应; 反应机理; 绿色化学

\section{Research Progress on Ammonia-Borane Based Direct Transfer Hydrogenation Reactions}

\author{
Yang, Xianghua* Xie, Zhenming $\mathrm{He}$, Jun Yu, Lin* \\ (School of Chemical Engineering and Light Industry, Guangdong University of Technology, Guangzhou 510006)
}

\begin{abstract}
Direct transfer hydrogenation reaction is a $\mathrm{H}$ transfer process from a hydrogen donor to a hydrogen acceptor without the presence of a catalyst - - the so-called metal-free reaction. It's an idea elementary process for mechanistic study, but was rarely reported due to very high energy barriers. However, it is revealed both theoretically and experimentally that increase in polarity of the substrates can efficiently lower the energy barrier, meaning that polar hydrogen donor and hydrogen acceptors might make such reactions happen. Ammonia borane is well-known as a chemical hydrogen storage material with very high $\mathrm{H}$ content. In the meanwhile, it's an ideal polar hydrogen donor containing both hydridic and protic $\mathrm{H}$ atoms. Applying ammonia borane as the polar hydrogen donor and some polar hydrogen acceptor molecules such as imines, polarized olefins, aldehydes and ketones, direct transfer hydrogenation reaction occurred under mild conditions. The reaction scope, similarity and differences in mechanism are reviewed herein.
\end{abstract}

Keywords ammonia borane; direct transfer hydrogenation; reaction mechanism; green chemistry

不饱和键的氢化是有机合成中最重要的基本反应 之一, 在已发展的多种氢化方法中, 转移氢化反应在均 相氢化领域获得了无与伦比的重要地位. 转移氢化反应 即从氢气以外的氢供体中转移两个氢原子到不饱和键 两端的反应, 由于能避免因直接使用氢气带来的潜在危 险和麻烦而备受青睐. 从机理的角度来讲, 转移氢化反 应可以分为两类 ${ }^{[1]}$ : 一类是氢原子先从氢供体转移到催 化剂(或一个非催化中心)上, 然后再转移给氢受体, 常 见的催化转移氢化反应都属于这种类型; 另一类则是两 个氢原子同步或先后从氢供体直接转移给氢受体, 即直
接转移氢化反应(Scheme 1).

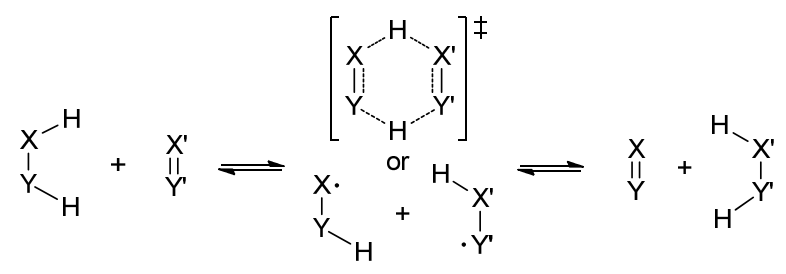

图式 1 双氢转移的基本过程 $\left(X, Y, X^{\prime}, Y^{\prime}\right.$ : 主族元素或过渡 金属)

Scheme 1 Elementary process of a double $\mathrm{H}$ transfer (X, Y, X' and $Y^{\prime}$ : main group element or transition metal)

*E-mail: yangxianghua@gdut.edu.cn,gych@gdut.edu.cn

Received December 31, 2014; revised February 14, 2015; published online February 28, 2015.

Project supported by the National Natural Science Foundation of China (Nos. 21407028, 21471037) and the Natural Science Foundation of Guangdong Province (No. 2014A030310449).

国家自然科学基金(Nos. 21407028, 21471037)以及广东省自然科学基金(No. 2014A030310449)资助项目. 
适用第一种机理的反应很多, 如早在 20 世纪初发 现的梅尔魏因一庞多夫一维尔来还原反应(MeerweinPonndorf-Verley 还原反应, 简称 MPV 还原 $)^{[2]}$, 就是以 异丙醇铝为催化剂通过一个协同的六元环过渡态先将 异丙醇中叔碳上的氢原子转移到羰基化合物中碳氧双 键的碳原子上, 再用异丙醇羟基上的氢原子取代铝原子 从而实现两个氢原子的转移; 若使用双功能催化剂, 如 Noyori 或 Shvo 型催化剂 ${ }^{[3]}$, 则会形成一个既有 $\mathrm{H}^{+}$又有 $\mathrm{H}^{-}$的金属氢化物中间体, 实现两个氢原子的同步转移.

适用第二类机理的反应很少, 最典型的例子为以乙 烷为氢供体、乙烯为氢受体的双氢转移 ${ }^{[4]}$, 理论计算的 结果表明可以经由一个 $4+2$ 对称性允许的协同过程发 生, 但反应能垒非常高 $(0 \mathrm{~K}$ 时 $297 \mathrm{~kJ} / \mathrm{mol})$, 因而很难实 现 $^{[5]}$; 若通过引入杂原子或推拉电子基团等方式把非极 性的乙烷和乙烯分别换成极性氢供体和含极性双键的 不饱和化合物, 则能使反应能垒有效降低 $(298 \mathrm{~K}$ 时 221 $\mathrm{kJ} / \mathrm{mol})^{[6]}$. 本文所述反应即以氨硼烷为极性氢供体、一 系列含极性双键的化合物为氢受体的直接转移氢化, 该 反应在温和条件甚至室温下就能有效发生.

氨硼烷 $\left(\mathrm{NH}_{3} \mathrm{BH}_{3}\right)$ 中氢元素的质量分数为 $19 \%$, 可有 效利用的氢含量(4 个 $\mathrm{H}$ 原子)也高达 $13 \%$, 高于 $\mathrm{NaBH}_{4}$ $(11 \% \mathrm{H}), \mathrm{LiAlH}_{4}(11 \% \mathrm{H}), \mathrm{BH}_{3}$-THF $(3 \% \mathrm{H})$ 等富氢分子, 且分子间双氢键的作用使之在常温常压下呈现为稳定 (含 $24 \% \mathrm{H}$ 的 $\mathrm{NH}_{4} \mathrm{BH}_{4}$ 极易分解)、无毒的白色固体(含 $21 \%$ $\mathrm{H}$ 的 $\mathrm{BH}_{3} \mathrm{BH}_{3}$ 为剧毒气体), 被视为理想的化学储氢材料 [7]. 作为化学储氢材料需要有效地释放和重新补充氢气 的途径, 因此研究者们的兴趣大多集中在氨硼烷的释氢 方面, 发展了包括热解、水解、过渡金属催化、纳米材料 催化等多种途径在内的高效脱氢方法 ${ }^{[8]}$. 由于 $\mathrm{H}$ 元素的 电负性刚好介于 $\mathrm{N}$ 和 $\mathrm{B}$ 元素之间 $(\mathrm{N}, \mathrm{H}$ 和 B 元素的电负 性分别为 $3.04,2.20$ 和 2.04), 使氨嗍烷中 $\mathrm{N}$ 和 B 所连的 氢原子分别呈正、负电性 ${ }^{[9]}$, 与催化转移氢化反应中最常 用的氢供体异丙醇同为极性富氢分子, 其在转移氢化反 应中作为氢供体的应用也逐渐被关 注 $^{[10]}$. 最新的如 Hartmann 等 ${ }^{[1]}$ 报道的以氨硼烷为氢源、 $\left[\mathrm{Pd}(\mathrm{NHC})\left(\mathrm{PR}_{3}\right)\right]$ (NHC: $N$-heterocyclic carbene, 即氮杂卡宾)为催化剂的烯 烃转移氢化反应. 以氨硼烷为氢供体的直接转移氢化反 应最早发现于 2010 年, 目前已被证明适用的底物, 包括 含有 $\mathrm{C}=\mathrm{N}$ 双键的亚胺类化合物、含有 $\mathrm{C}=\mathrm{C}$ 双键的极化 烯烃以及含有 $\mathrm{C}=\mathrm{O}$ 双键的醛、酮类化合物, 对于这些反 应的机理已有实验和理论计算等多方面的研究结果, 本 文将对这些反应分别进行介绍.

\section{1 亚胺类化合物的直接转移氢化反应}

\section{1 亚胺的直接转移氢化}

2010 年, Berke 课题组 ${ }^{[12]}$ 在研究以氨嗍烷为氢供体的
亚胺催化转移氢化反应过程中，意外发现在没有催化剂 参与时反应效率反而更高, 于是有了第一例成功在温和 条件下发生的非催化转移氢化反应: 亚胺化合物在 $60{ }^{\circ} \mathrm{C}$ 氮气保护的四氢呋喃(THF)溶液中从氨硼烷分子夺 取 2 个氢原子生成相应胺类化合物，产率接近 $100 \%$ ，而 反应时间则依取代基的不同从半小时到几天不等(表 1 ).

表 1 亚胺与氨嗍烷在 THF 中的反应

Table 1 Reactions of $\mathrm{NH}_{3} \mathrm{BH}_{3}$ with various imines in THF

\begin{tabular}{|c|c|c|c|c|c|}
\hline $\mathrm{R}$ & $\mathrm{R}$ & Time $^{a} / \mathrm{h}$ & $\mathrm{R}$ & $\mathrm{R}$ & $\operatorname{Time}^{a} / \mathrm{h}$ \\
\hline $\mathrm{Ph}$ & $\mathrm{Ph}$ & 7 & $\mathrm{Cy}$ & $t-\mathrm{Bu}$ & 21 \\
\hline $\mathrm{Ph}$ & $\mathrm{CH}(\mathrm{Ph})_{2}$ & 24 & $\mathrm{Ph}_{2}$ & $\mathrm{H}$ & 14 \\
\hline $\mathrm{MeOC}_{6} \mathrm{H}_{4}$ & $\mathrm{Ph}$ & 8 & $\mathrm{Ph}$ & $\mathrm{MeOC}_{6} \mathrm{H}_{4}$ & 24 \\
\hline $\mathrm{ClC}_{6} \mathrm{H}_{4}$ & $\mathrm{Ph}$ & 5 & $\mathrm{Ph}$ & $\mathrm{ClC}_{6} \mathrm{H}_{4}$ & 4 \\
\hline $\mathrm{NO}_{2} \mathrm{C}_{6} \mathrm{H}_{4}$ & $\mathrm{Ph}$ & 3 & $\mathrm{Ph}$ & $\mathrm{NO}_{2} \mathrm{C}_{6} \mathrm{H}_{4}$ & 0.5 \\
\hline $\mathrm{MeOC}_{6} \mathrm{H}_{4}$ & $\mathrm{MeOC}_{6} \mathrm{H}_{4}$ & 20 & $\mathrm{ClC}_{6} \mathrm{H}_{4}$ & $\mathrm{MeOC}_{6} \mathrm{H}_{4}$ & 20 \\
\hline $\mathrm{MeOC}_{6} \mathrm{H}_{4}$ & $\mathrm{ClC}_{6} \mathrm{H}_{4}$ & 5 & $\mathrm{ClC}_{6} \mathrm{H}_{4}$ & $\mathrm{ClC}_{6} \mathrm{H}_{4}$ & 4 \\
\hline
\end{tabular}

${ }^{a}$ Times needed for reactions to complete (conversion $>99 \%$ ).

\section{2 反应机理}

为深入了解这一反应的机理，包括理论计算、同位 素跟踪技术、同位素效应以及取代基效应在内的多种研 究方法被采用. 为了检验这个反应与乙烷和乙烯的双氢 转移模型之间的相似性，他们首先采用了理论计算的手 段. 两种不同方法计算的结果都表明不管从热力学还是 动力学角度, 协同的双氢转移过程都具有最低的反应能 垒. 而后同位素追踪的结果表明此反应具有很好的位置 选择性(Scheme 2): 氨嗍烷( $\mathrm{AB}$ )中富电子的硼接氢原子 $\mathrm{H}_{\mathrm{B}}$ 转移到了亚胺 $\mathrm{C}=\mathrm{N}$ 中电负性较小的碳原子上, 缺电 子的氮接氢原子 $\mathrm{H}_{\mathrm{N}}$ 则转移到了该极性双键中电负性较 强的氮原子上, 符合异电性相吸的趋势. 采用亚胺大量 过量从而人为造就准一级反应条件下测得的氞元素的 动力学同位素效应(Duterium Kinetic Isotope Effect, DKIE)结果表明(Scheme 2), $\mathrm{H}_{\mathrm{N}}$ 的氛代产生了正同位素 效应 $\left(k_{\mathrm{H}} / k_{\mathrm{D}}>1\right)$, 即氢原子被氛取代后反应速率有所减 慢 $\left(k_{\mathrm{AB}} / k_{\mathrm{A}(\mathrm{D}) \mathrm{B}}=1.93, k_{\mathrm{AB}(\mathrm{D})} / k_{\mathrm{A}(\mathrm{D}) \mathrm{B}(\mathrm{D})}=1.61\right) ; \mathrm{H}_{\mathrm{B}}$ 的氛代则 产生了反同位素效应 $\left(k_{\mathrm{H}} / k_{\mathrm{D}}<1\right)$, 即重原子取代后反应 速率反而加快 $\left(k_{\mathrm{AB}} / k_{\mathrm{AB}(\mathrm{D})}=0.87, k_{\mathrm{A}(\mathrm{D}) \mathrm{B}} / k_{\mathrm{A}(\mathrm{D}) \mathrm{B}(\mathrm{D})}=0.72\right)$. 介于 $0.7 \sim 2$ 之间且不等于 1 的 $k_{\mathrm{H}} / k_{\mathrm{D}}$ 值通常意味着直接 连接重原子的键并未在决速步中发生断裂, 在本反应中 可以理解为 $\mathrm{B}-\mathrm{H}$ 和 $\mathrm{N}-\mathrm{H}$ 键在决速步发生了部分断 裂. 根据莫尔斯的势能曲线理论(图 1), 正同位素效应的 产生通常是由于过渡态的势能曲线比基态的平缓, 反同 位素效应则刚好相反 ${ }^{[13]} . \mathrm{D}_{\mathrm{N}}$ 产生的正同位素效应可解 释为过渡态中部分断裂和部分生成的键强度近似，此时 原子间相对较弱的结合势必使其势能曲线变得平缓(图 
1 , 左); $\mathrm{D}_{\mathrm{B}}$ 产生的反同位素效应则可解释为由于在过渡 态中部分形成了比 $\mathrm{B}-\mathrm{H}$ 键(键能约 $389 \mathrm{~kJ} / \mathrm{mol}$ ) 更强的 C $-\mathrm{H}$ 键(键能约 $414 \mathrm{~kJ} / \mathrm{mol}$ ), 从而导致其过渡态势能曲 线更加陡峭 (图 1, 右). 而过渡态中 $\mathrm{N} \cdots \mathrm{H} \cdots \mathrm{N}$ 和 $\mathrm{B} \cdots \mathrm{H} \cdots \mathrm{C}$ 的存在既表明反应很可能经由协同的双氢转 移机理，又能很好地解释反应的位置选择性.

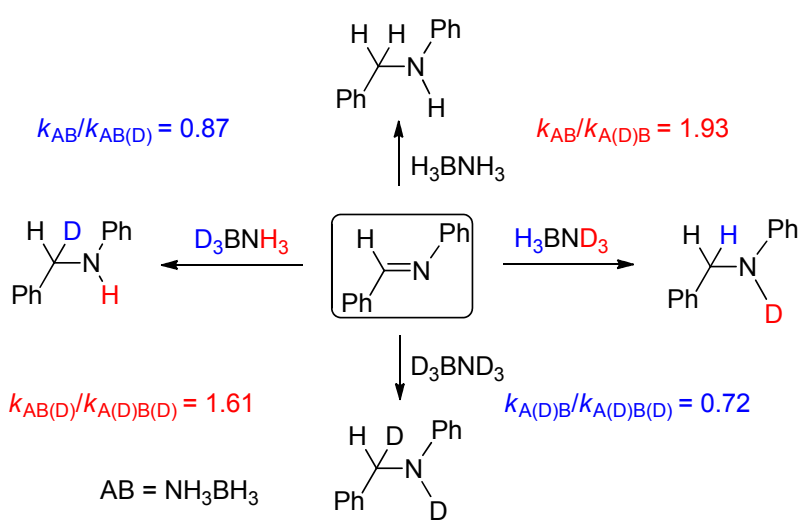

图式 2 亚胺与氨跀烷的直接转移氢化反应过程中的位置选 择性与氛动力学同位素效应

Scheme 2 Regiospecificity \& DKIE in the direct transfer hydrogenation process between imine and $\mathrm{NH}_{3} \mathrm{BH}_{3}$

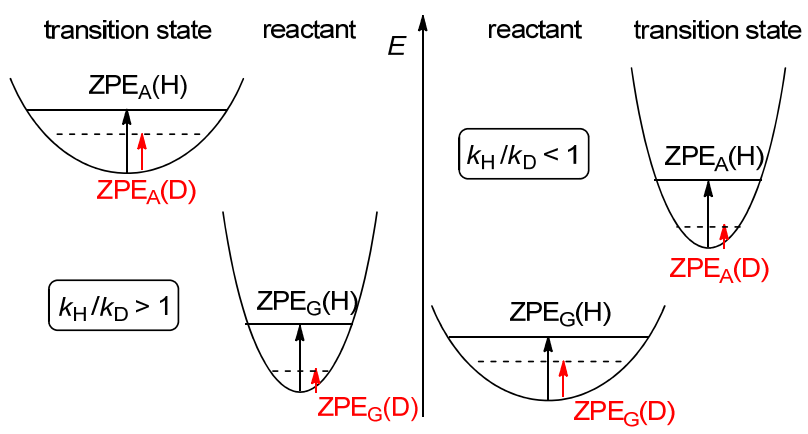

图 1 莫尔斯势能曲线示意图

Figure 1 The Morse potential curve

Left: normal DKIE, right: inverse DKIE. Where ZPE for zero point energy, subscripts $\mathrm{G}$ for ground state and $\mathrm{A}$ for activated state.

最后还对 $\mathrm{C}=\mathrm{N}$ 双键上 $\mathrm{C}$ 端和 $\mathrm{N}$ 端分别连接的苯环 对位上取代基不同时的反应速率常数基于 Hammett 方 程进行了拟合 ${ }^{[14]}$, 得到两种情况下的 Hammett 图形斜率 $\rho$ 均大于零 $(\mathrm{C}$ 端 $\rho=0.69, \mathrm{~N}$ 端 $\rho=1.61)$, 即吸电子能力 强的取代基团能加快反应速率, 这表明在过渡态中 $\mathrm{C}=$ $\mathrm{N}$ 双键上不管是 $\mathrm{C}$ 原子还是 $\mathrm{N}$ 原子上的电子密度都有 所增加，也很好地与协同的反应机理相吻合一一若该反 应中的两个氢原子系分步转移, 负电性的 $\mathrm{H}_{\mathrm{B}}$ 和正电性 的 $\mathrm{H}_{\mathrm{N}}$ 势必造成相应中心原子上相反的电子密度变化. 总之, 理论与实验的结果均指向一个结论: 极性氢供体 氨嗍烷与极性不饱和化合物亚胺之间的转移氢化反应 不需要催化剂的参与就能在温和条件下发生, 而且经由 协同的双氢同步转移机理.

\section{2 极化烯烃的转移氢化}

\section{1 极化烯烃的直接转移氢化反应}

2011 2012 年, Berke 课题组 ${ }^{[15]}$ 相继发表两篇氨硼 烷与双键一端被两个强极性基团取代的烯烃之间的直 接转移氢化反应. 与亚胺的直接转移氢化比起来, 这些 极化烯烃与氨嗍烷之间的反应更加温和——在乙腈中 室温下甚至更低的温度下就能很快发生反应(在 THF 中 则更快), 且转化率高达 99\%(表 2). 不过这一反应对底 物的极性要求较高, 必需在双键一端的碳上接两个强吸 电子性质的取代基，若该碳原子上只接了一个吸电基团 或双键两端碳原子上都接了吸电基团则无反应活性或 发生很复杂的反应.

\section{表 2 极化烯烃与氨硼烷在乙腈中的反应 ${ }^{a}$}

Table 2 Reactions of $\mathrm{NH}_{3} \mathrm{BH}_{3}$ with various polarized olefins in acetonitrile

\begin{tabular}{|c|c|c|c|}
\hline $\mathrm{R}, \mathrm{R}^{\prime}$ & $E_{-} E^{\prime}$ & Time & Yield $^{b} / \%$ \\
\hline$-\left(\mathrm{CH}_{2}\right)_{5-}$ & $\mathrm{CN}, \mathrm{CN}$ & $1 \mathrm{~h}$ & 99 \\
\hline$-\left(\mathrm{CH}_{2}\right)_{5^{-}}$ & $\mathrm{CN}, \mathrm{CO}_{2} \mathrm{Me}$ & $2 \mathrm{~h}$ & 99 \\
\hline$-\left(\mathrm{CH}_{2}\right)_{4^{-}}$ & $\mathrm{CN}, \mathrm{CN}$ & $5 \mathrm{~h}$ & 99 \\
\hline $\mathrm{Me}, \mathrm{Me}$ & $\mathrm{CN}, \mathrm{CN}$ & $4 \mathrm{~h}$ & 99 \\
\hline $\mathrm{Me}, t$-Bu & $\mathrm{CN}, \mathrm{CN}$ & $1 \mathrm{~h}$ & 99 \\
\hline $\mathrm{Et}, \mathrm{Ph}$ & $\mathrm{CN}, \mathrm{CN}$ & $6 \mathrm{~h}$ & 99 \\
\hline $\mathrm{H}, \mathrm{H}$ & $\mathrm{CN}, \mathrm{H}$ & $2 \mathrm{~d}$ & Trace \\
\hline $\mathrm{CN}, \mathrm{CN}$ & $\mathrm{CN}, \mathrm{CN}$ & $10 \mathrm{~min}$ & Complex \\
\hline
\end{tabular}

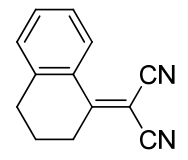

$10 \mathrm{~h}$

99

$\begin{array}{lll}\mathrm{CN}, \mathrm{CO}_{2} \mathrm{Me} & 0.5 \mathrm{~h} & 99\end{array}$

$\mathrm{Ph}, \mathrm{H} \quad \begin{array}{lll}\mathrm{CO}_{2} \mathrm{Me}, & 3 \mathrm{~d} & 98\end{array}$

$\mathrm{Ph}, \mathrm{H} \quad \mathrm{CN}, \mathrm{H} \quad 10 \mathrm{~d} \quad$ Trace

$\mathrm{Ph}, \mathrm{Ph} \quad \mathrm{CN}, \mathrm{CN} \quad 5 \mathrm{~d} \quad 92$

9-Floren $\quad \mathrm{CN}, \mathrm{CN} \quad 2 \mathrm{~d} \quad 98$

$\overbrace{\mathrm{CN}}^{\mathrm{CN}} 2 \mathrm{~d} \quad 85^{\circ}$

(1d $86^{c}$

${ }^{a}$ The reaction in THF is even faster, which made it difficult to understand the mechanism; ${ }^{b}$ Reactions monitored by in situ ${ }^{1} \mathrm{H}$ NMR and conversion confirmed by GC-MS; ${ }^{c}$ Only the double bond directly connected to two $\mathrm{CNs}$ being hydrogenated.

\section{2 反应机理}

双键两端分别被环己烷基和两个腈基取代的极化 烯烃因反应活性适中被选中用于反应机理的探讨. 虽然 
与亚胺同为极性不饱和化合物, 极化烯烃与氨硼烷之间 的反应却被证明经由不同的反应机理. 一方面, 同位素 追踪的结果表明此反应也具有很好的位置选择性 (Scheme 3): 氨硼烷中富电子的硼接氢原子 $\mathrm{H}_{\mathrm{B}}$ 转移到了 烯烃的双键中电子密度较小的烷基端碳原子上, 缺电子 的氮接氢原子 $\mathrm{H}_{\mathrm{N}}$ 则转移到了该极性双键中连接两个吸 电基团的碳原子上, 依然符合异电性相吸的趋势. 另一 方面，DKIE 却有不同的结果(Scheme 3), $\mathrm{D}_{\mathrm{N}}$ 依然产生 了正同位素效应 $\left(k_{\mathrm{AB}} / k_{\mathrm{A}(\mathrm{D}) \mathrm{B}}=1.55, k_{\mathrm{AB}(\mathrm{D})} / k_{\mathrm{A(D)B(D)}}=1.62\right)$; $\mathrm{D}_{\mathrm{B}}$ 则几乎没有反同位素效应 $\left(k_{\mathrm{H}} / k_{\mathrm{D}} \approx 1\right)$, 即氛代后反应 速率几乎没有任何改变 $\left(k_{\mathrm{AB}} / k_{\mathrm{AB}(\mathrm{D})}=1.00, k_{\mathrm{A}(\mathrm{D}) \mathrm{B}} / k_{\mathrm{A}(\mathrm{D}) \mathrm{B}(\mathrm{D})}\right.$ $=1.04)$, 这样的同位素效应, 表明只有 $\mathrm{N}-\mathrm{H}$ 键的断裂 参与了决速步, $\mathrm{B}-\mathrm{H}$ 键的断裂则完全未参与决速步, 即 很可能发生在决速步之前或者之后.

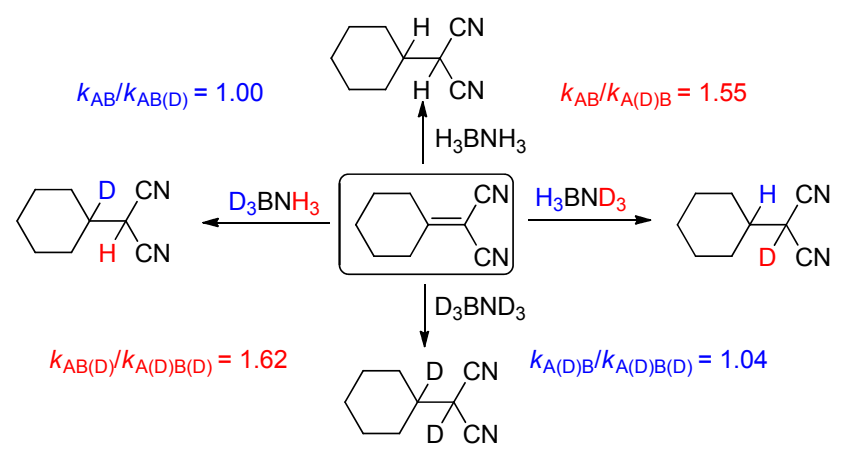

图式 3 极性烯烃与氨嗍烷的直接转移氢化反应过程中的位 置选择性与氞动力学同位素效应

Scheme 3 Regiospecificity \& DKIE in the direct transfer hydrogenation process between polarized olefin and $\mathrm{NH}_{3} \mathrm{BH}_{3}$

凭借该烯烃与氨嗍烷在 THF 中超高的反应活性, 开展了以 $\mathrm{THF}$ 为溶剂的低温反应并用原位核磁实时监 测以捕捉可能的反应中间体. 如 Scheme 4 所示, 一个嗍 氢化反应的中间体在 $-40{ }^{\circ} \mathrm{C}$ 被检测到, 且其后续反应 能很好地与室温下的反应相吻合, 即在有过量烯烃存在 时能与另一分子烯烃反应生成两分子相应烷烃和氨硼 烷脱两分子氢的产物环硼氮烷(borazine, BZ), 而无自由 烯烃存在时则直接生成一分子烷烃和氨嗍烷脱一分子 氢的三聚产物环硼氮六烷(cyclotriborazane, CTB). 该中 间体的存在进一步证明氨硼烷中 $\mathrm{H}_{\mathrm{B}}$ 的转移在决速步之 前已经完成, 只有 $\mathrm{H}_{\mathrm{N}}$ 的转移发生在决速步. 通过对烯 烃双键烷基端接一个氢原子一个苯环时苯环对位取代 基变化时反应速率常数的改变, 该反应的 Hammett 拟合 图形为斜率接近 1 的直线, 意味着决速步的反应中心是 一个负电荷积累即电子密度增大的过程. 从捕捉到的中 间体结构来看, 决速步为 $\mathrm{H}_{\mathrm{N}}$ 的转移, 反应中心则为连 接两个 $\mathrm{CN}$ 和 $\mathrm{BH}_{2} \mathrm{NH}_{3}$ 的碳原子, 此时, 电负性比碳小 的嗍原子接到碳上自然会使其电子密度比双键状态时
有所增大.

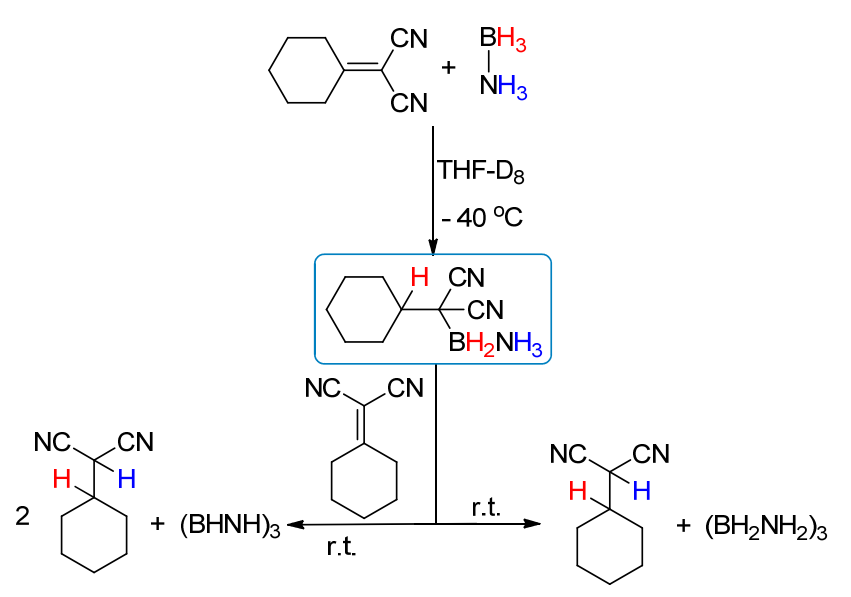

图式 4 利用低温核磁捕获的嗍氢化产物中间体及其反应性 能

Scheme 4 A hydroboration intermediate trapped by low temperature NMR and its reactivity

为了揭示氨硼烷的脱氢偶合路径, 该课题组又开展 了数个实验, 如往反应混合物中加入环己烯, 因为环己 烯与氨嗍烷之间的直接反应在室温下很慢, 因此其硼氢 化产物 $\mathrm{Cy}_{2} \mathrm{BNH}_{2}$ 的生成可以用于验证反应过程中活性 中间体 $\left[\mathrm{NH}_{2} \mathrm{BH}_{2}\right]$ 的生成 ${ }^{[16]}$ 一在上述反应液中加入环 己烯后很快就检测到了 $\mathrm{Cy}_{2} \mathrm{BNH}_{2}$, 表明此过程中生成了 氨硼烷脱一分子氢的中间产物 $\left[\mathrm{NH}_{2} \mathrm{BH}_{2}\right]$. 为了鉴别最 终的脱氢偶合产物 $\mathrm{CZ}$ 的形成是经由 $\mathrm{CTB}$ 还是通过 $\left[\mathrm{NH}_{2} \mathrm{BH}_{2}\right]$ 直接脱一分子氢并聚合, 又检验了 $\mathrm{CTB}$ 与极 性烯烃之间的直接反应, 结果发现 CTB 的直接转移氢 化反应活性远远不如氨硼烷, 从而证明实际反应过程中 第一步的脱氢产物 $\left[\mathrm{NH}_{2} \mathrm{BH}_{2}\right]$ 并未先三聚成 CTB 再进一 步参与转移氢化反应, 而是继续与一分子烯烃反应给出 一分子氢再三聚生成 CZ. 综合上述机理研究的结果可 以得到一个结论: 极性氢供体氨硼烷与极化烯烃间的直 接转移氢化反应在室温下就能快速发生, 但不是经由协 同的双氢同步转移路径, 而是通过先转移 $\mathrm{H}_{\mathrm{B}}$ 、决速步再 转移 $\mathrm{H}_{\mathrm{N}}$ 的分步双氢转移途径.

\section{3 醛、酮类化合物的转移氢化}

\section{1 醛、酮与氨硼烷之间的嗍氢化反应及机理}

从结构上看, 含碳氧双键的醛、酮类化合物与亚胺 的结构非常类似，因而在与氨硼烷的反应性能方面也被 期待有相似的反应活性. 2011 年 Berke 课题组 ${ }^{[17]}$ 率先发 现了醛、酮等含羰基化合物与氨硼烷之间的反应并推测 了可能的机理, 他们发现醛、酮类化合物与氨硼烷在非 质子溶剂如 THF 中发生的是硼氢化反应而非预期的直 接转移氢化反应, 而且以酮为底物的反应需要加热才能 
完成, 醛的反应则可以在室温下完成; 在甲醇等质子溶 剂中, 由于嗍氢化产物直接发生了醇解, 可以在室温下 快速得到相应的饱和醇(Scheme 5).

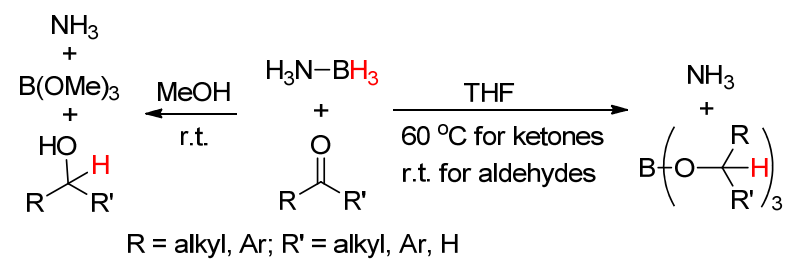

图式 5 氨嗍烷与醛、酮类化合物在质子溶剂和非质子溶剂中 的反应

Scheme 5 Reaction of $\mathrm{NH}_{3} \mathrm{BH}_{3}$ with aldehydes/ketones in protic and aprotic solvents

二苯甲酩被选取用于该硼氢化反应的机理研究. 首 先是采用被选择性氛代的氨硼烷与其反应, 并通过核磁 氢谱、碳谱以及気谱的检测来追踪気的去向. 结果表明 嗍上氢原子的氞代即 $\mathrm{D}_{\mathrm{B}}$ 原子转移到了羰基的碳原子上, 而 $\mathrm{D}_{\mathrm{N}}$ 则以游离 $\mathrm{ND}_{3}$ 的形式存在于反应液中. 而后动力 学同位素效应的结果又表明不管是 $\mathrm{D}_{\mathrm{B}}$ 还是 $\mathrm{D}_{\mathrm{N}}$ 都产生了 较小的正同位素效应且相比较而言, $\mathrm{D}_{\mathrm{N}}$ 的影响更大 $\left(k_{\mathrm{AB}} /\right.$ $k_{\mathrm{AB}(\mathrm{D})}=1.28, \quad k_{\mathrm{AB}} / k_{\mathrm{A}(\mathrm{D}) \mathrm{B}}=1.74, \quad k_{\mathrm{A}(\mathrm{D}) \mathrm{B}} / k_{\mathrm{A}(\mathrm{D}) \mathrm{B}(\mathrm{D})}=1.10$, $\left.k_{\mathrm{AB}(\mathrm{D})} / k_{\mathrm{A}(\mathrm{D}) \mathrm{B}(\mathrm{D})}=1.49\right)$, 这可以解释为氨硼烷中氨的离去 与 $\mathrm{H}_{\mathrm{B}}$ 的转移是一个同步且协同的过程, 两者都在决速 步中发生, 或者由于氨砳烷中双氢键的存在导致氨的离 去较难, 因而氨的离去就是决速步, 所以 $\mathrm{D}_{\mathrm{N}}$ 对反应速 率的影响反而比 $\mathrm{D}_{\mathrm{B}}$ 更大.

为了进一步检验以氨嗍烷为嗍氢化试剂的反应与 普通的硼氢化反应之间的相似性, 另一种硼氢化试剂 $\mathrm{BH}_{3} \cdot \mathrm{THF}$ 被用于与二苯甲酮反应, 此时硼氢化反应在 室温下就很快分两步发生, 先很快生成二烷氧基硼烷, 而后缓慢生成相应的三烷氧基嗍烷 ${ }^{[18]}$. 由于 $\mathrm{BH}_{3}$ 与 $\mathrm{THF}$ 之间没有双氢键的存在因而比起氨硼烷更容易游离出 自由的 $\mathrm{BH}_{3}$, 以 $\mathrm{BH}_{3} \cdot \mathrm{THF}$ 为硼氢化试剂的反应更容易发 生, 但最终的硼氢化产物却与以氨硼烷为硼氢化试剂的 最终产物完全一致, 进一步验证了该过程为硼氢化反应 的可靠性. 当把在 THF 中完全转化为硼氢化产物的反 应混合物置于 $-80{ }^{\circ} \mathrm{C}$ 并监测 ${ }^{11} \mathrm{~B}$ NMR 时, 发现其可以 可逆的转化为另外一种四配位硼的化合物, 后来被证明 是该嗍氢化产物在低温下与溶剂 THF 之间的配合物. 结合上述机理研究的结果以及文献[19]中关于硼氢化反 应机理的推测, Berke 课题组得出的结论是, 该硼氢化反 应的决速步为氨硼烷中 $\mathrm{BH}_{3}$ 与 $\mathrm{NH}_{3}$ 的解离. 该过程有可 能经由 Scheme 6 中 $\mathrm{A}$ 或者 $\mathrm{B}$ 路径, 路径 $\mathrm{A}$ 是直接解离, 路径 B 则是氨硼烷解离的同时结合了一分子底物, 即近 似于 $\mathrm{S}_{\mathrm{N}} 2$ 取代的过程. 考虑到低温的原位反应中并没有 检测到相关的中间体, 因而推测路径 $\mathrm{A}$ 的可能性更大.
而后的嗍氢化反应过程则很可能依次经由三个协同的 四元环过渡态逐级反应直至形成最终产物.

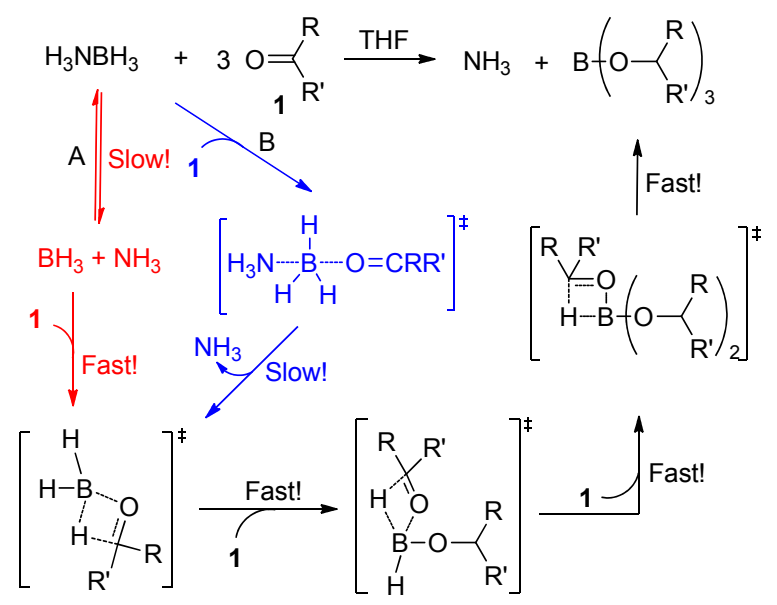

图式 6 氨嗍烷与醛、酮类化合物之间的硼氢化反应可能机理 Scheme 6 Possible mechanism for the hydroboration of ketones and aldehydes with $\mathrm{NH}_{3} \mathrm{BH}_{3}$

\section{2 醛与氨硼烷之间的直接转移氢化反应及机理}

2012 年, 大连化物所陈萍课题组与焚红军课题 组 ${ }^{[20]}$ 研究发现醛类化合物与氨硼烷在非质子溶剂中发 生的并非上述的硼氢化反应, 而是直接转移氢化反应, 而且与亚胺的直接转移氢化反应经由同样的双氢同步 转移机理(Scheme 7). 他们以物质的量比为 $1: 1$ 的氨硼 烷和醛的 THF 溶液在室温下惰性气体氛围中反应，通 过原位的 FT-IR 检测到了与羰基的消耗同步增长的羟基 峰, ${ }^{1} \mathrm{H}$ NMR 和 ${ }^{13} \mathrm{C}$ NMR 谱图都能验证相应醇的生成, 而且对不同对位取代的苯甲醛都具有很好的适用性.

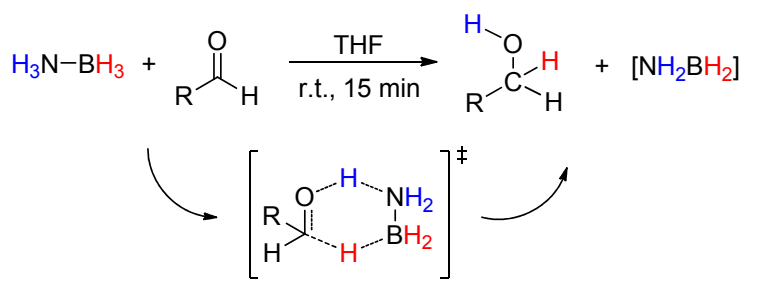

图式 7 氨硼烷与醛之间的直接转移氢化反应及可能机理 Scheme 7 Direct transfer hydrogenation of aldehydes with $\mathrm{NH}_{3} \mathrm{BH}_{3}$ and possible mechanism

他们以苯甲醛为特征底物对这个反应的机理进行 了探讨，首先采用部分氛代的氨硼烷与之反应并检测产 物中気的位置, 发现 $\mathrm{D}_{\mathrm{B}}$ 转移到了羰基的碳原子上, $\mathrm{D}_{\mathrm{N}}$ 则转移到了羰基的氧原子上，而非 Berke 报道的以游离 $\mathrm{ND}_{3}$ 的形式存在. 进一步的反应动力学研究以及分子模 拟的结果表明, 当两种反应物的物质的量比为 $1: 1$ 时, 该反应的速率与氨硼烷和醛的浓度均呈一级相关, 总反 应则为二级反应. 在相同条件下测得的氛动力学同位素 效应的结果表明，不管是 $\mathrm{D}_{\mathrm{N}}$ 还是 $\mathrm{D}_{\mathrm{B}}$ 都有较大的正同位 
素效应 $\left(k_{\mathrm{AB}} / k_{\mathrm{AB}(\mathrm{D})}=2.85, k_{\mathrm{AB}} / k_{\mathrm{A}(\mathrm{D}) \mathrm{B}}=3.47\right)$, 说明两种氢 原子的转移都参与了决速步, 进一步论证了协同的双氢 同步转移机理的可能性.

此外, 他们还发现氨硼烷的锂/钠/钻盐能与亚胺或酮 在更温和的条件下发生直接转移氢化反应且经由双氢转 移机理, 理论计算结果表明该过程的决速步为金属氢化 物的离去 ${ }^{[21]}$. 若底物为醛, 发生的则是硼氢化产物 ${ }^{[20]}$.

2013 年辽宁师范大学周丹红课题组与大连化物所 牀红军课题组 ${ }^{[22]}$ 合作发表了一篇理论计算的文章, 其 中对以氨嗍烷为氢供体的直接转移氢化反应机理进行 了比较全面的探讨, 他们认为不光亚胺与氨硼烷之间的 转移氢化是协同的双氢同步转移机理, 上述的醛或酮与 氨硼烷之间的转移氢化或硼氢化反应过程也应经由同 样的机理. 因为亚胺或醛的双氢同步转移机理前面已经 提过, 这里主要介绍他们计算的酮与氨硼烷之间发生硼 氢化反应的双氢同步转移机理. 他们先是计算了 Berke 课题组提出分步反应的机理, 即先发生氨硼烷解离然后 转移 $\mathrm{H}_{\mathrm{B}}$ 的反应途径的可行性, 发现此过程中的决速步 也就是能垒最高的步骤为 $\mathrm{H}_{\mathrm{B}}$ 的转移 $(169.0 \mathrm{~kJ} / \mathrm{mol})$ 而非 氨的解离 $(140.5 \mathrm{~kJ} / \mathrm{mol})$. 考虑到 $169.0 \mathrm{~kJ} / \mathrm{mol}$ 的能垒已 经很高, 他们又提出了一种新的反应机理(Scheme 8), 即第一步是协同的双氢同步转移生成相应的醇并释放 出的活性中间体 $\left[\mathrm{NH}_{2} \mathrm{BH}_{2}\right]$, 而后 $\left[\mathrm{NH}_{2} \mathrm{BH}_{2}\right]$ 马上与醇发 生醇解反应并生成硼氢化的产物直至生成最后产物. 此 过程的决速步则为第一步的双氢转移过程 (146.0 $\mathrm{kJ} / \mathrm{mol}),\left[\mathrm{NH}_{2} \mathrm{BH}_{2}\right]$ 的醇解则仅需 $97.4 \mathrm{~kJ} / \mathrm{mol}$ 的能垒, 均 远低于之前的分步反应机理, 事实上该结论也很好的与 之前的实验数据相吻合.

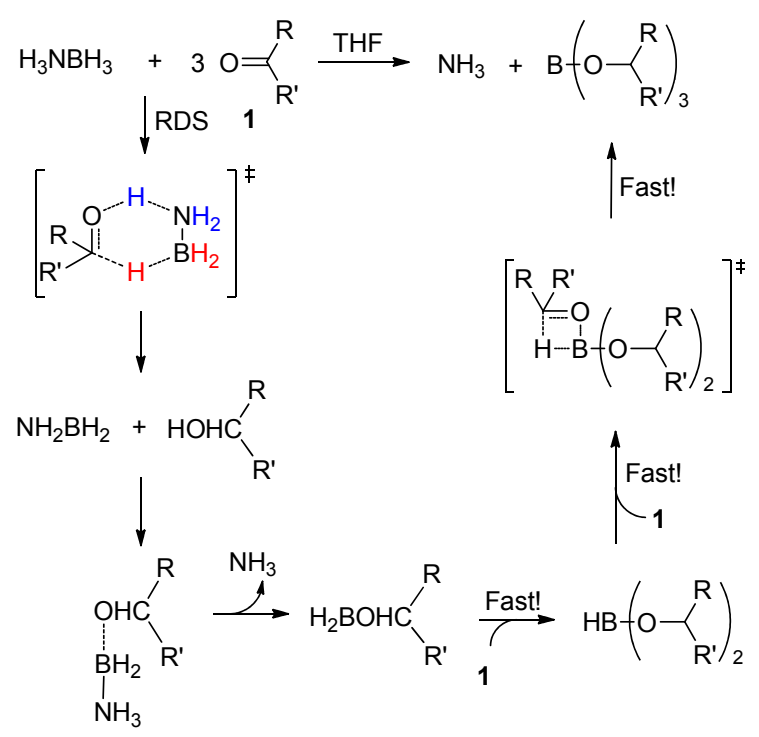

图式 8 氨嗍烷与酮的嗍氢化反应的直接转移氢化反应机理 Scheme 8 A direct transfer hydrogenation pathway for the hydroboration of ketones with $\mathrm{AB}$

\section{3 含羰基化合物与氨硼烷之间反应的机理探讨}

虽然都是经由协同的双氢同步转移过程，但同为含 羰基的极性不饱和化合物醛、酮与氨硼烷的反应却被认 为生成了截然不同的两种产物一一直接转移氢化产物 和嗍氢化产物, 若细想却也能找出其中的相似之处: (1) 可以确定的是氨硼烷硼上的氢原子转移到了羰基碳上, 关于氮上氢原子的去向，陈萍课题组认为转移到了羰基 氧上、Berke 课题组则认为以游离氨的形式存在. 事实 上, 原料中的氨基氢和产物中的羟基氢或游离氨上的氢 全是活泼氢原子，其在核磁等检测结果中有一定的不确 定性, 游离氨和羟基在红外光谱中的出峰位置也有一定 相似性. 硼氢化产物和直接转移氢化产物的确认主要是 通过核磁谱图或红外光谱上 $\mathrm{OH}$ 或游离 $\mathrm{NH}_{3}$ 的存在, 而 这两者的峰形非常相似且哪怕是在同一溶剂中都有可 能随环境的细微变化而改变出峰位置. (2)从核磁硼谱看 两者也有一定的相似之处: 除了原料氨硼烷之外, 都只 有 $\delta 19$ 处也就是嗍氢化产物有出峰, 并没有出现其余的 氨硼烷脱氢偶合产物. 陈萍课题组以硼氢化产物的峰很 低为理由认为这只是副产物, Berke 课题组却以没有其 他嗍物种的存在为理由认为嗍氢化产物是唯一产物. 仔 细比对可以发现两个课题组所采用的氨硼烷与羰基化 合物的物质的量的比分别 $1: 1$ 和 $1: 2$ (或 $1: 3$ ), 也就 是说前者中氨硼烷处于过量三分之二的情形而后者中 氨硼烷仅过量三分之一或反应完全，在氨硼烷大量过量 时完全有可能出现三配位的硼氢化产物硼谱出峰低的 情况, 加大氨嗍烷的消耗程度应能明显改善. (3)从氛的 动力学同位素效应结果来看, 虽然数值上差异较大, 不 过两者得到的结果都是正同位素效应且 $\mathrm{D}_{\mathrm{N}}$ 的影响比 $\mathrm{D}_{\mathrm{B}}$ 要大, 意味着不管是 $\mathrm{H}_{\mathrm{B}}$ 还是 $\mathrm{H}_{\mathrm{N}}$ 的氛代都会使反应速率 减慢, 即很可能其所连接的化学键在决速步中发生了全 部或部分断裂. 之所以数值上会有这么大的差异, 很重 要的一个原因是陈萍课题组以 $1: 1$ 的物质的量之比(氨 硼烷: 醛)也就是在二级反应的情况下测的醛的转化速 率, 而 Berke 课题组是以 $1: 5$ 的物质的量之比也就是准 一级反应的情况下测的氨硼烷的消耗速率.

如此来看, 这两种反应结论的得出实际上基于非常 类似的实验事实. 不过值得一提的是, Berke 课题组在机 理研究中选用的代表性化合物是二苯甲酮, 也就是说对 于醛的反应仅有前期的反应数据而没有深入研究醛的 反应机理, 其在没有数据支撑的情况下把酲类化合物归 入硼氢化反应的结论中可以认为不够严谨.

\section{4 结论}

总之，极性氢供体氨硼烷与极性不饱和化合物之间 的直接转移氢化反应是一类新颖而绿色的反应, 为氨硼 
烷的利用开辟了新的领域. 对于这些反应的机理研究有 利于从分子层次了解反应过程, 能为氨硼烷相关反应中 廉价(绿色)催化剂的设计提供科学指导, 进而实现弱极 性或非极性烯烃、炔烃的氢化, 并为氨硼烷中氢原子的 利用尤其是氢气的可控释放提供更多可能途径.

\section{References}

[1] (a) Berke, H. ChemPhysChem 2010, 11, 1837.

(b) Ujaque, G.; Lledós, A. J. Mol. Struct.: THEOCHEM 2009, 903, 123.

[2] (a) Meerwein, H.; Schmidt, R. Justus Liebigs Ann. Chem. 1925, 444, 221.

(b) Ponndorf, W. Angew. Chem. 1926, 39, 138.

(c) Verley, A. Bull. Soc. Chim. Fr. 1925, 37, 871.

[3] (a) Noyori, R.; Okhuma, T.; Kitamura, M.; Takaya, H.; Sayo, N.; Kumobayashi, H.; Akuragawa, S. J. Am. Chem. Soc. 1987, 109, 5856.

(b) Noyori, R. In Organic Synthesis, Wiley-Interscience, John Wiley \& Sons, New York, 1994, pp. 16 94 and references cited therein.

(c) Blum, Y.; Czarkie, D.; Rahamim, Y.; Shvo, Y. Organometallics 1985, 4, 1459.

(d) Shvo, Y.; Czarkie, D.; Rahamim, Y.; Chodosh, D. F. J. Am. Chem. Soc. 1986, 108, 7400.

(e) Casey, C. P.; Strotman, N. A.; Beetner, S. E.; Johnson, J. B.; Priebe, D. C.; Vos, T. E.; Khodavandi, B.; Guzei, I. A. Organometallics 2006, 25, 1230.

[4] (a) Miller, D. J.; Smith, D. M.; Chan, B.; Radom, M. Mol. Phys. 2006, 104, 777.

(b) Fernández, I.; Sierra, M. A.; Cossío, F. P. J. Org. Chem. 2007, $72,1488$.

[5] Feller, D. F.; Schmidt, M. W.; Ruedenberg, K. J. Am. Chem. Soc. 1982, 104, 960.

[6] McKee, M. L.; Stanbury, D. M. J. Am. Chem. Soc. 1992, 114, 3214.

[7] (a) Hamilton, C. W.; Baker, R. T.; Staubitz, A.; Manners, I. Chem. Soc. Rev. 2009, 38, 279.

(b) Marder, T. B. Angew. Chem., Int. Ed. 2007, 46, 8116.

(c) Gutowska, A.; Li, L.; Shin, Y.; Wang, M.; Li, X. S.; Linehan, J. C.; Smith, R. S.; Kay, B. D.; Schmid, B.; Shaw, W.; Gutowski, M.; Autrey, T. Angew. Chem., Int. Ed. 2005, 44, 3578.

(d) Grochala, W.; Edwards, P. P. Chem. Rev. 2004, 104, 1283.

[8] (a) Zhang, L.; Tu, Q.; Chen, X.; Liu, P. Prog. Chem. 2014, 26, 749。 (b) Yang, L.; Cao, N.; Du, C.; Dai, H.; Hu, K.; Luo, W.; Cheng, G. Mater. Lett. 2014, 115, 113.

(c) Li, H.; Yang, Q,; Chen, X.; Shore, S. G. J. Organomet. Chem.
2014, 751, 60 .

(d) Kim, Y.; Baek, H.; Lee, J. H.; Yeo, S.; Kim, K.; Hwang, S. J.; Eun, B.; Nam, S. W.; Lim, T. H.; Yoon, C. W. Phys. Chem. Chem. Phys. 2013, 15, 19584.

(e) Hartmann, C. E.; Jurcik, V.; Songis, O.; Cazin, C. S. Chem. Commun. 2013, 49, 10629.

(f) Marder, T. Angew. Chem., Int. Ed. 2007, 46, 8116.

(k) Denney, M. C.; Pons, V.; Hebden, T. J.; Heinekey, D. M.; Goldberg, K. I. J. Am. Chem. Soc. 2006, 128, 12048.

[9] (a) Parvanov, V. M.; Schenter, G. K.; Hess, N. J.; Daemen, L. L.; Hartl, M.; Stowe, A. C.; Camaionia, D. M.; Autrey, T. Dalton Trans. 2008, 33, 4514.

(b) Rasul, G. K. G.; Prakash, S.; Olah, G. A. THEOCHEM 2007, 818,65 .

[10] (a) Yurderi, M.; Bulut, A.; Zahmakiran, M.; Gulcan, M.; Ozkar, S. Appl. Catal. B, Environ. 2014, 160, 534.

(b) Dong, H.; Berke, H. J. Organomet. Chem. 2011, 696, 1803.

(c) Jiang, Y.; Blacque, O.; Fox, T.; Frech, C. M.; Berke, H. Organometallics 2009, 28, 5493.

(d) Jiang, Y.; Berke, H. Chem. Commun. 2007, 3571.

[11] Hartmann, C. E.; Jurcik, V.; Songis, O.; Cazin, C. S. Chem. Commun. 2013, 49, 1005.

[12] Yang, X.; Zhao, L.; Fox, T.; Wang, Z.-X.; Berke, H. Angew. Chem., Int. Ed. 2010, 49, 2058.

[13] Morse, P. M. Phys. Rev. 1929, 34, 57.

[14] (a) Hammett, L. P. J. Am. Chem. Soc. 1937, 59, 96. (b) Hammett, L. P. Chem. Rev. 1935, 17, 125.

[15] (a) Yang, X.; Fox, T.; Berke, H. Chem. Commun. 2011, 47, 2053. (b) Yang, X.; Fox, T.; Berke, H. Org. Biomol. Chem. 2012, 10, 852.

[16] (a) Smythe N. C.; Gordon, J. C. Eur. J. Inorg. Chem. 2010, 509. (b) Pons, V.; Baker, R. T.; Szymczak, N. K.; Heldebrant, D. J.; Linehan, J. C.; Matus, M. H.; Grant D. J.; Dixon, D. A. Chem. Commun. 2008, 6597.

(c) Shrestha, R. P.; Diyabalanage, H. V. K.; Semelsberger, T. A.; Ott K. C.; Burrell, A. K. Int. J. Hydrogen Energy 2009, 34, 2616.

[17] Yang, X.; Fox, T.; Berke, H. Tetrahedron 2011, 7121.

[18] Brown, H. C.; Schlesinger, H. I.; Burg, A. B. J. Am. Chem. Soc. 1939, 61, 673.

[19] (a) Brown, H. C.; Wang, K, K.; Chandrasekharan, J. J. Am. Chem. Soc. 1983, 105, 2343.

(b) Kudo, T.; Higashide, T.; Ikedate, S.; Yamataka, H. J. Org. Chem. 2005, 70, 5157.

[20] Xu, W.; Fan, H.; Wu, G.; Chen, P. New J. Chem. 2012, 36, 1496.

[21] Xu, W.; Wu, G.; Yao, W.; Fan, H.; Wu, J.; Chen, P. Chem. Eur. J. 2012, 18, 13885 .

[22] Wang, X.; Yao, W.; Zhou, D.; Fan, H. Mol. Phys. 2013, 111, 3014. 